Instituto Internacional de Investigación y Desarrollo Tecnológico Educativo INDTEC, C.A.

DOI: https://doi.org/10.29394/Scientific.issn.2542-2987.2020.5.15.19.362-383

OAI-PMH: http://www.indteca.com/ojs/index.php/Revista Scientific/oai

Ensayo Original / Original Essay

\title{
Aportes de las reformas curriculares a la educación obligatoria en el Ecuador
}

\author{
Autores: Miguel Ángel Herrera Pavo \\ Universidad Andina Simón Bolívar, UASB \\ miguel.herrera.p@uasb.edu.ec \\ Quito, Ecuador \\ https://orcid.org/0000-0002-0321-7235 \\ María Gladys Cochancela Patiño \\ Universidad Nacional de Educación, UNAE \\ maria.cochancela@unae.edu.ec \\ Azogues, Ecuador \\ https://orcid.org/0000-0001-8845-9710
}

\section{Resumen}

Las reformas curriculares de la educación obligatoria en los últimos años en el Ecuador han incidido, fundamentalmente, en el enriquecimiento de la estructura de las propuestas, cuya creciente complejidad ha tratado de dar respuesta a las necesidades de la acción docente; en la redefinición progresiva de lo que entendemos por contenidos de la enseñanza, y en la delimitación de las responsabilidades de construcción del currículo, como medio de atender la diversidad de escenarios educativos del país. El presente trabajo analiza a partir de estos elementos la evolución de las diferentes reformas curriculares del país, específicamente, las del año 1996, 2010 y 2016, consignado los principales aportes con los que estas reformas han contribuido a la educación del país, entre los que se cuentan unas mejores condiciones para la atención de la diversidad, unas orientaciones curriculares concebidas para el enriquecimiento del acto educativo, una definición de los contenidos acorde con un enfoque de educación integral, y la delegación de responsabilidades en la construcción del currículo a los equipos docentes para una mejor contextualización de los proceso de enseñanza y aprendizaje.

Palabras clave: reforma educativa; planes educativos; diversificación de la educación.

\section{Cómo citar este ensayo:}

Herrera, M., \& Cochancela, M. (2020). Aportes de las reformas curriculares a la educación obligatoria en el Ecuador. Revista Scientific, 5(15), 362-383, e-ISSN: 2542-2987. Recuperado de: https://doi.org/10.29394/Scientific.issn.2542-2987.2020.5.15.19.362-383

Fecha de Recepción: 03-10-2019
Fecha de Aceptación:

30-12-2019
Fecha de Publicación: 05-02-2020 


\title{
Contributions of curricular reforms to compulsory education in Ecuador
}

\begin{abstract}
The curricular reforms of compulsory education in recent years in Ecuador have influenced, fundamentally, the enrichment of the structure of the proposals, whose increasing complexity has tried to respond to the needs of the teaching action; in the progressive redefinition of what we understand by the contents of education, and in the delimitation of the responsibilities of curriculum construction, as a means of addressing the diversity of educational scenarios in the country. The present work analyzes from these elements the evolution of the different curricular reforms of the country, specifically, those of the year 1996, 2010 and 2016, consigned the main contributions with which these reforms have contributed to the education of the country, among which there are better conditions for the attention of diversity, curricular orientations conceived for the enrichment of the educational act, a definition of the contents in accordance with a comprehensive education approach, and the delegation of responsibilities in the construction of the curriculum to the teaching teams for a better contextualization of the teaching and learning process.
\end{abstract}

Keywords: educational reform; educational plans; diversification of education.

How to cite this essay:

Herrera, M., \& Cochancela, M. (2020). Contributions of curricular reforms to compulsory education in Ecuador. Revista Scientific, 5(15), 362-383, e-ISSN: 2542-2987. Recovered from: https://doi.org/10.29394/Scientific.issn.2542-2987.2020.5.15.19.362-383

Date Received:

03-10-2019
Date Acceptance:

30-12-2019
Date Publication: 05-02-2020 


\section{Introducción}

El currículo puede considerarse en palabras de Bolaños y Molina (1990): como el conjunto de experiencias que viven los y las estudiantes con la guía del docente y el objetivo de alcanzar determinadas finalidades educativas (pág. 34); es, según Perlo (2019): "un proyecto pedagógico amplio y complejo, que involucra una concepción del ser, de la realidad y la naturaleza del encuentro humano" (pág. 70); una expresión de intereses políticos, culturales y económicos que responde a tensiones globales, nacionales y locales y que, además, debe abordar la tensión entre reproducción y transformación social. A este respecto, Sánchez (2018), expresa que: "el concepto contemporáneo de currículo queda definido como acuerdo político y como selección consciente de contenidos" (pág. 54).

Por otro lado, Pascual (2001), destaca que: "en este sentido, es difícil encontrar que algún Estado moderno renuncie a participar, aunque sea en una expresión limitada, en la construcción del currículum escolar" (pág. 38). Como comenta Gimeno (2010): "a través de ese proyecto institucional se expresan fuerzas, intereses o valores y preferencias de la sociedad, de determinados sectores sociales, de las familias, de grupos políticos..." (pág. 30).

En el Ecuador, como documentan Poveda, Díaz, Abendaño, Benalcazar, Araujo y Arboleda (1994): el Estado ha intervenido en la definición de los contenidos de la enseñanza desde la creación del Ministerio de Instrucción Pública en el año 1884, a través del Consejo Superior, y la posterior promulgación de la Ley Orgánica de Instrucción Pública del año 1906 (pág. 2); aunque no es hasta la reforma del año 1996 que las propuestas curriculares oficiales comienzan a ser en algo más que un mero programa de estudios.

En este sentido, como nos dice Coll (2006a): si bien, uno de los cambios fundamentales que suelen realizar las reformas educativas, vinculado con nuevos escenarios sociales, culturales, económicos, científicos y tecnológicos, está relacionado con los contenidos de la enseñanza (pág. 6); hoy en día existe 
un consenso, como menciona Stabback (2016): con respecto a que el currículo debe ir más allá de este proceso, constituyéndose en una guía sobre lo que queremos hacer y cómo debemos hacerlo (pág. 9); e introduciendo orientaciones y guías sobre la metodología, los recursos y la evaluación que permitan llevar la propuesta diseñada por el Estado a las aulas.

A este respecto, otro de los elementos fundamentales de la política curricular será la definición del margen que tienen los actores del sistema educativo, las comunidades educativas, de adaptar las propuestas que vienen del nivel central a sus intereses e inquietudes, resignificándola, apropiándosela, en lo que Morelli (2019), define como: "la tensión entre la hegemonía y la autonomía para la traducción y recontextualización de sus discursos" (pág. 11); para atacar lo que Díaz (2015), denomina: "ausencia de significado" (pág. 354).

En el presente ensayo nos enfocaremos en: 1). Analizar cómo las propuestas curriculares del Ecuador se han ido complejizando y diversificando; 2). Cómo ha ido evolucionando el concepto de contenido curricular; y 3). Cómo se han concebido las responsabilidades en el diseño de las sucesivas propuestas.

Atendiendo al primero de los objetivos, analizaremos los componentes y la estructura de las propuestas curriculares, y cómo estos atienden a conceptualizaciones específicas del acto educativo, evolucionando desde los clásicos listados de contenidos apropiados para un sistema basado en la transmisión de contenidos, hasta las actuales propuestas que abordan todas las facetas de la actividad del aula con materiales curriculares y didácticos complementarios.

En cuanto a la conceptualización de los contenidos de la enseñanza, hemos visto cómo se han complejizado, evolucionando con la concepción misma de la enseñanza, desde enfoques tradicionales transmisivos y memorísticos, que contemplaban exclusivamente contenidos conceptuales 0 
cognitivos, hacia contenidos más complejos que se esfuerzan por incluir componentes de tipo procedimental y actitudinal más acordes con enfoques socio-constructivistas de la enseñanza, incluyendo, además, la preocupación por la realización, es decir, por determinar la forma en que se supone que esos contenidos de la enseñanza deben ser movilizados por los estudiantes en otros contextos, distintos del educativo.

En este sentido, actualmente, como sugiere Coll (2006b): "los contenidos y competencias identificados como básicos con el fin de justificar su presencia en el currículo escolar remiten siempre a la realización de unos aprendizajes considerados necesarios para los alumnos" (pág. 7); en función de lo que la sociedad espera de ellos. Estas expectativas de la sociedad con respecto a las generaciones futuras han favorecido lo que Coll (2006c): define como "lógica esencialmente acumulativa" (pág. 5); que sobrecarga las propuestas curriculares con contenidos de la más diversa índole, no solo a partir de la actualización de conocimientos de las áreas curriculares clásicas, sino también agregando nuevas asignaturas y temas transversales que responden a nuevas necesidades sociales.

Una de las propuestas para abordar el problema de la sobrecarga de contenidos en los currículos, desarrollada por Coll (2006d): consiste en establecer una distinción entre contenidos básicos imprescindibles que serían aquellos que "condicionan o determinan negativamente el desarrollo personal y social" (pág. 8); de los estudiantes en caso de no adquirirlos, y deseables, constituidos por "aprendizajes que pueden ser "recuperados" sin grandes dificultades más allá del término de la educación obligatoria" (pág. 8).

Por último, la cuestión de quién debe diseñar las propuestas curriculares también ha sido un tema de amplio debate, entre modelos donde el Estado no interviene y delega a las instituciones educativas la responsabilidad de diseñar currículos que logren alcanzar criterios mínimos con respecto a los logros de aprendizaje que se recogen en estándares 
educativos nacionales, hasta modelos completamente intervencionistas que dictan a las instituciones educativas y a los docentes los planes de estudio con el más mínimo detalle, sin dejarles espacio para la toma de decisiones relacionadas con su contexto.

Ante este escenario, los enfoques socio-constructivistas proponen una responsabilidad distribuida entre los distintos niveles de toma de decisiones del sistema educativo. Así, como recoge Kreisel (2016): el Estado diseña una propuesta general, que es revisada y enriquecida por la institución educativa con un proyecto curricular institucional, que posteriormente el docente puede adaptar a la realidad de su aula mediante planificaciones y proyectos específicos; definiéndose tres niveles de concreción curricular sucesivos que permiten que las propuestas curriculares se adapten a los intereses y necesidades específicas de una comunidad educativa y de un grupo de estudiantes, mediante un proceso dialógico (pág. 5).

A continuación, revisaremos estos aspectos en las diferentes propuestas curriculares que se han sucedido en el Ecuador durante las últimas dos décadas.

\section{Desarrollo}

Tres momentos marcan la evolución del currículo en el Ecuador, las reformas del año 1996, 2010 y 2016; previamente, los diferentes gobiernos solo elaboraron programas de estudio que proporcionaban listas de contenidos y que no podrían ser considerados como currículos en su acepción actual. La primera reforma introdujo el concepto de destrezas en el currículo ecuatoriano, con la intención de distanciarse de los modelos previos basados en la transmisión de contenido netamente conceptual o cognitivo y con la perspectiva de una educación más integral; además, esta reforma abordó, por primera vez, el tratamiento de contenidos transversales en el currículo. La segunda profundizó en el concepto de destrezas, agregándoles criterios de 
desempeño que permitieran delimitar las expectativas con respecto a su realización, amplió el tratamiento de temas transversales y agregó indicadores para la evaluación; además, a partir de este nuevo currículo, por primera vez en el país se diseñaron estándares de aprendizaje. Finalmente, siguiendo las recomendaciones de Coll (2006e): la tercera reforma, hoy vigente, introdujo el concepto de aprendizajes básicos (pág. 8); a través de una estructura de bloques y áreas curriculares, vinculó las intenciones educativas con criterios e indicadores de evaluación, y a estos con unos nuevos estándares para la evaluación externa. A pesar de estos importantes hitos en el diseño curricular de nuestro país, las tres reformas fallaron en el propósito de reducir los contenidos de aprendizaje. A continuación, revisaremos en mayor detalle las peculiaridades de cada una de ellas.

\subsection{La reforma curricular del año 1996}

En el año 1996, según recoge el trabajo de Calvas (2010): nace la Reforma Curricular Consensuada para la Educación Básica. El Consejo Nacional de Educación y el Ministerio de Educación y Cultura pusieron en marcha el proceso de reforma curricular del año 1996 a partir del análisis de la fallida reforma curricular con enfoque conceptual del año 1994, propuesta que recibió fuertes críticas de los docentes del país (págs. 80-87); esta nueva reforma quería distinguirse, adaptándose a la realidad ecuatoriana, con el objetivo de mejorar la calidad de la educación. Para ello, se diseñó un sistema de validación con un triple enfoque: técnico, disciplinar y pedagógico, en el que participaron expertos nacionales y extranjeros, involucrando a los docentes del sistema nacional, tanto de educación básica, como de los institutos pedagógicos de formación de profesorado y de las facultades de educación; por ello recibió el apelativo de consensuada.

Entre las características más relevantes de esta reforma se cuentan la ausencia formal de un enfoque pedagógico, la incorporación a la educación 
básica de la educación preescolar como obligatoria, y la introducción de las nociones de destrezas y valores en el currículo. En cuanto a este último punto, el cambio introducido responde a intención de distanciarse de un currículo basado en contenidos de corte conceptual o cognitivo, dando los pasos necesarios para una educación integral que incluyera no solo los clásicos contenidos academicistas, sino también procedimientos y actitudes.

La reforma consensuada, como recoge la propuesta emitida por Consejo Nacional de Educación y el Ministerio de Educación, Cultura, Deportes y Recreación (1997): estableció por primera vez 10 años de educación obligatoria, estructurados a través de áreas de conocimiento (en un inicio se desarrollan las de Lenguaje y comunicación, Matemática, Entorno natural y social, Ciencias naturales, y Estudios sociales; posteriormente, de forma gradual, se desarrollaron también las áreas de Cultura estética, Cultura física, y Lengua extranjera) y ejes transversales (inicialmente, Educación práctica de valores, Interculturalidad y Educación ambiental, a los que posteriormente se fueron agregando otros, como Coeducación, gracias a los aportes de organizaciones sociales, como el Consejo Nacional de la Mujer), y dejando una amplia autonomía a los docentes para su desarrollo en el aula. Para cada una de las áreas, se establecen consideraciones generales a modo de lineamientos, destrezas fundamentales organizadas por años, contenidos generales y mínimos por temas y año, y recomendaciones metodológicas generales (págs. 1-114). Como podemos ver, la gran ausencia de esta reforma es la evaluación, que solo se contempla en las recomendaciones metodológicas generales en algunas áreas aludiendo a las ideas de evaluación integral, formativa e innovadora, pero sin ninguna concreción. Esta falencia se abordaría en la siguiente propuesta curricular.

\subsection{La reforma curricular del año 2010}

Según indica el Ministerio de Educación (2010a): la evaluación de la 
reforma de 1996 permitió comprender las dificultades que experimentaron los docentes en su aplicación, entre las que se encontraban la imprecisión en la definición de los contenidos de enseñanza, la dificultad de comprender las destrezas, la carencia de criterios e indicadores de evaluación y la ausencia de articulación con el nivel de bachillerato (pág. 8).

Ante este análisis y con el objetivo de actualizar la reforma del año 1996 en cuanto a sus proyecciones social, científica y pedagógica, la Actualización y Fortalecimiento Curricular de la Educación General Básica se plantea como una propuesta estructurada para el segundo nivel de concreción curricular que, a cambio de limitar la flexibilidad del instrumento y la autonomía de instituciones educativas y docentes en la selección y secuenciación de las intenciones educativas y en el diseño de la intervención, ofrece una estructura más sólida, riqueza en orientaciones metodológicas que pretenden contribuir a la competencia profesional docente, y un sistema de indicadores de evaluación por área y año, que se complementará posteriormente con la formulación de los estándares de aprendizaje en el año 2012.

La nueva propuesta entraría en vigor en el año 2009 mediante acuerdo ministerial nro. 0611-09 para las áreas de Lenguaje y comunicación, Matemática, Estudios sociales y Ciencias naturales. En el caso del área de Educación Física, hubo que esperar hasta el año 2012, mientras que el currículo de Cultura Estética del año 1997 se mantuvo vigente.

En esta propuesta, según se recoge en el documento emitido por el Ministerio de Educación (2010b): el Buen Vivir, principio constitucional basado en el Sumak Kawsay, se tomó como principio rector de la transversalidad del currículo, incorporando 5 ejes temáticos: la "Interculturalidad" (pág. 16); la "formación de una ciudadanía democrática" (pág. 17); la "protección del medioambiente" (pág. 17); el "cuidado de la salud y de los hábitos de recreación de los estudiantes" (pág. 17); y la "educación sexual en los jóvenes" (pág. 17). 
La propuesta curricular afirma basarse, según el Ministerio de Educación (2010c): en principios de la "pedagogía crítica" (pág. 9); el "desarrollo de la condición humana y preparación para la comprensión" (pág. 9); "un pensamiento y modo de actuar lógico, crítico y creativo" (pág. 10); el "aprendizaje productivo y significativo" (pág. 11); el "desarrollo de destrezas con criterios de desempeño (pág. 11); el "empleo de tecnologías de la información y la comunicación" (pág. 12); y la "evaluación integradora de los resultados de aprendizaje" (págs. 12-13). Por otro lado, presenta una gran cantidad de elementos, como se puede visualizar en la figura 1, para los que no establece una relación explícita, de este modo, por ejemplo, no es posible ver la correspondencia entre las intenciones educativas y los indicadores de evaluación, lo que dificulta establecer el logro de los objetivos planteados y del propio perfil de salida.

Figura 1. Estructura de la propuesta curricular del año 2010.

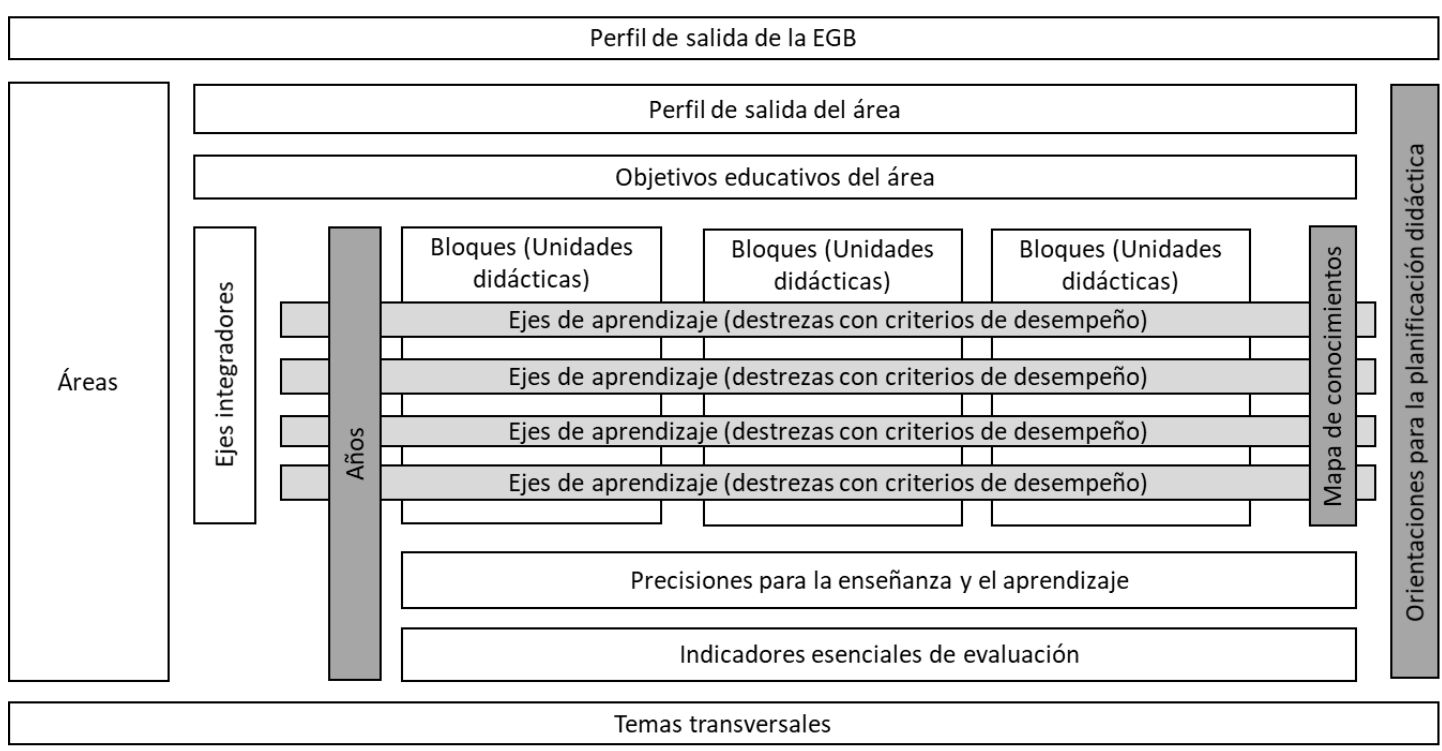

Fuente: Los Autores (2019).

Además, este currículo, que se presenta como una propuesta mesocurricular, realmente llega a definir la estructura de las unidades didácticas 
bajo el nombre de bloques curriculares, estableciendo, por tanto, directrices normativas que corresponden al tercer nivel de concreción curricular. De este modo, la propuesta curricular del año 2010 invade las que debieran ser las competencias de las instituciones educativas y de los propios docentes en cuanto al desarrollo del currículo, limitando tanto la flexibilidad del instrumento, como la posibilidad de diálogo del este con el contexto social y cultural de la institución educativa. Esta concepción de la norma curricular relega a instituciones educativas y docentes al papel de meros reproductores de una propuesta establecida de manera vertical.

Por otro lado, esta propuesta curricular generó una adaptación para las escuelas pluridocentes con la intención de facilitar el trabajo de estas escuelas, que suponen un porcentaje importante de las escuelas del país, en ella se hace un trabajo de selección y agrupación de destrezas con criterios de desempeño, y se diseña un instrumento llamado Plan de trabajo simultáneo que pretende organizar el trabajo del aula. Las falencias en cuanto estructura, flexibilidad y evaluación serían abordadas en el siguiente reajuste curricular.

\subsection{La reforma curricular del año 2016}

Entre mayo del año 2011 y noviembre del año 2012, se realizó un monitoreo encaminado a revelar el grado de implantación de la reforma realizada en el año 2010, y entre octubre y noviembre del año 2013 se lleva a cabo una investigación denominada, El uso y percepciones del currículo de Educación General Básica. Ambos insumos determinan un ajuste a la propuesta curricular vigente hasta el momento. Este ajuste curricular se realizará a través del proyecto, Fortalecer la calidad educativa a través del ajuste curricular y de los estándares de aprendizaje, con la colaboración de la Organización Estados Iberoamericanos.

El punto de partida de la nueva reforma, según el Ministerio de Educación (2016a): consiste en la definición de un perfil de salida para la 
educación obligatoria, al que se denominó, Perfil de salida del bachiller ecuatoriano, producto de un proceso colaborativo en el que participaron los principales actores de la comunidad educativa y del sector productivo (pág. 7). Este perfil se alimenta a partir de las intenciones educativas que emanan del marco normativo vigente y establece el horizonte que los estudiantes deben alcanzar a partir del trabajo en las diferentes áreas del aprendizaje presentes en la propuesta curricular.

Este primer paso destaca la relevancia que para la nueva propuesta tiene la definición de las intenciones educativas, que se concretan, a partir del perfil de salida, en objetivos integradores para cada subnivel educativo, generales para cada área, y específicos para cada área en cada uno de los subniveles, en función de los cuales se seleccionarán y secuenciarán los contenidos de la propuesta para cada área y subnivel educativo.

El nuevo currículo, por tanto, al igual que el anterior, estará organizado por áreas de conocimiento, pero hará una propuesta simplificada en cuanto a la estructura, y el máximo nivel de desagregación de la propuesta se corresponderá con el subnivel educativo (Preparatoria, 1 año, Elemental, 3 años, Media, 3 años, y superior, 3 años, en el nivel Educación Básica, y el nivel de Bachillerato, 3 años), como se muestra en la figura 2.

Cada una de las áreas se desarrolla a través de bloques curriculares que agrupan los contenidos con un razonamiento epistemológico. Estos bloques inician en el primer año de la educación obligatoria y terminan en el último año del bachillerato. Esta estructura permite que los contenidos puedan moverse dentro del bloque, a través de los diferentes niveles y subniveles educativos, en función de las necesidades de cada contexto educativo específico. Esta propuesta de flexibilización se apoya en la clasificación de los contenidos en dos grandes grupos, tal y como sugirió Coll (2006f): imprescindibles y deseables (pág. 8); y en una completa articulación entre los cuatro subniveles de la educación básica y el nivel de bachillerato. 
Figura 2. Estructura de la propuesta curricular del año 2016 (Currículo de los niveles de educación obligatoria).

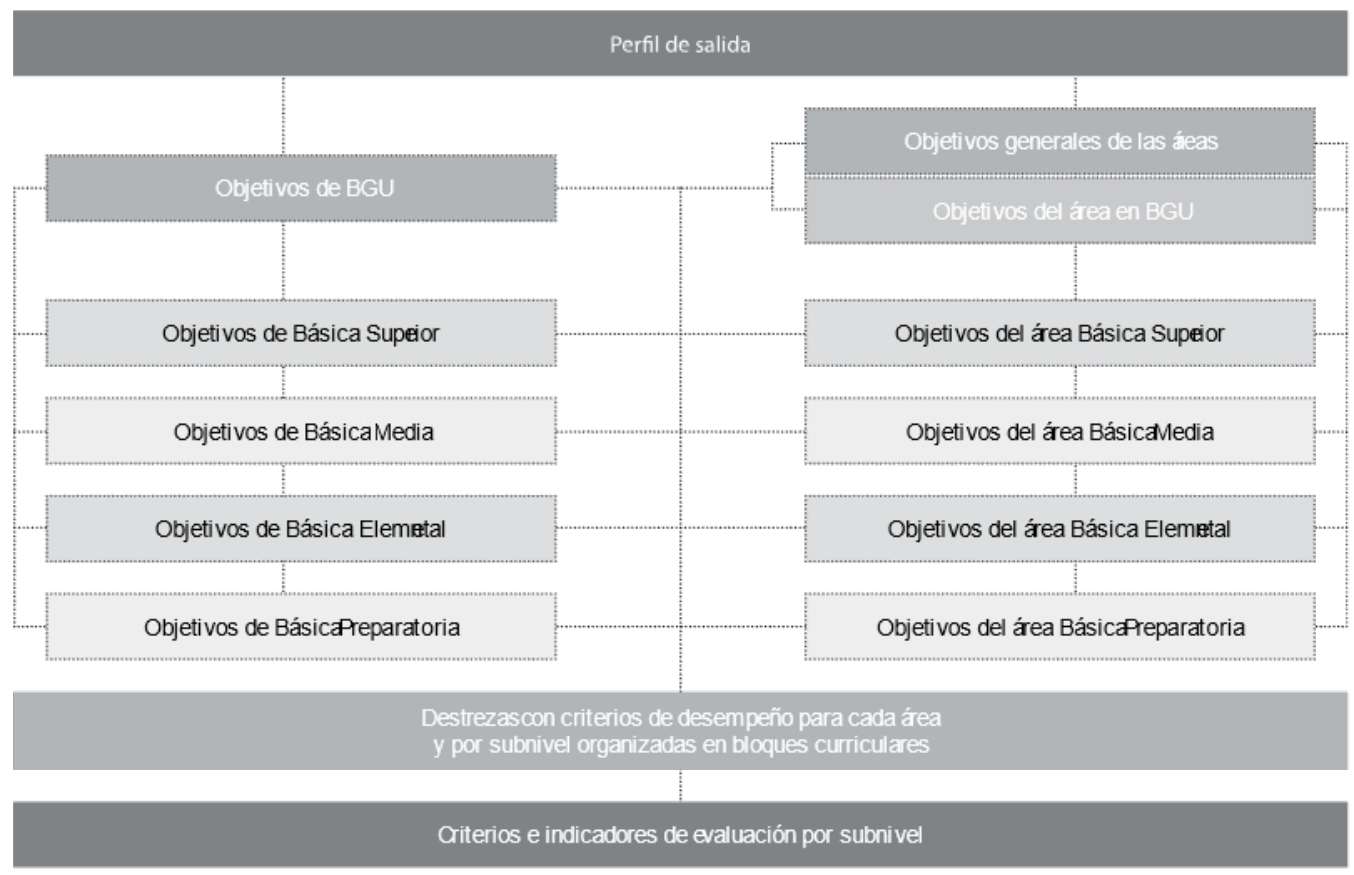

Fuente: Ministerio de Educación (2016b).

Los contenidos del currículo del año 2016, al igual que en la reforma del año 2010, se definen como destrezas con criterios de desempeño, y engloban conocimientos conceptuales, cognitivos, procedimentales y actitudinales que se circunscriben a un contexto determinado de realización, delimitando la forma en que se espera que los estudiantes puedan emplearlos.

Por otro lado, el currículo del año 2016, hace una propuesta de secuenciación de los contenidos de cada subnivel en función de los criterios de evaluación. La evaluación toma entonces gran relevancia, y los criterios se acompañan de indicadores para la evaluación y de sugerencias metodológicas para su desarrollo. En esta ocasión, los estándares de aprendizaje para la evaluación externa se hacen coincidir con los indicadores de evaluación del currículo, si bien, como apuntan Delgado, Vera, Cruz y Pico (2018): los 
primeros apuntan a la evaluación de productos, mientras que los segundos están además centrados en los procesos (pág. 62). Esta articulación de la evaluación se realiza para impedir que la evaluación externa influencie o incluso desvíe los procesos en el aula de las intenciones educativas explicitadas en el currículo.

El borrador de la propuesta, construido por especialistas disciplinares y curriculares nacionales y extranjeros, fue sometido a un riguroso proceso de validación que trató de llevar el debate sobre el currículo a todos los rincones del país, involucrando a todas las zonas y a 139 de los 140 distritos educativos del nivel desconcentrado del sistema educativo ecuatoriano, expresado en la tabla 1.

Tabla 1. Talleres de validación de la propuesta curricular del año 2016.

\begin{tabular}{|c|c|c|c|}
\hline Zonas & Lugares & Distritos & Asistentes \\
\hline Zona 1 & $\begin{array}{l}\text { Esmeraldas, Lago } \\
\text { Agrio, Ibarra, San } \\
\text { Lorenzo, Tulcán }\end{array}$ & 16 & 545 \\
\hline Zona 2 & Coca, Latacunga & 9 & 201 \\
\hline Zona 3 & $\begin{array}{l}\text { Ambato, Riobamba } \\
\text { Puyo, Tena }\end{array}$ & 15 & 414 \\
\hline Zona 4 & $\begin{array}{l}\text { Manta, Portoviejo, } \\
\text { Santo Domingo }\end{array}$ & 16 & 275 \\
\hline Zona 5 & $\begin{array}{l}\text { Babahoyo, Quevedo, } \\
\text { Milagro }\end{array}$ & 14 & 301 \\
\hline Zona 6 & Azogues, Macas & 17 & 175 \\
\hline Zona 7 & $\begin{array}{l}\text { Loja, Machala, } \\
\text { Saraguro, Zamora }\end{array}$ & 19 & 368 \\
\hline Zona 8 & Guayaquil & 22 & 206 \\
\hline Zona 9 & Quito & 11 & 243 \\
\hline 9 zonas & 26 ciudades & 139 distritos & 2728 participantes \\
\hline
\end{tabular}

Fuente: Los Autores (2019).

La socialización, tal y como consta en un informe interno del Ministerio de Educación (2015a): fue realizada en los meses de julio, agosto, septiembre y octubre del año 2015, y contó con la participación de grupos de docentes y 
directivos de todo el país (593 de la especialidad de Matemática, 522 de la especialidad de Lengua y Literatura, 451 de la especialidad de Ciencias Naturales, 430 de Ciencias Sociales, 322 especialistas de Educación Física, 246 de Educación Cultural y Artística, 164 especialistas en el nivel de Preparatoria), que contribuyeron con sus aportes al proceso de construcción de los documentos curriculares (pág. 3).

Como resultado de este proceso, como se aprecia en el citado informe del Ministerio de Educación (2015b): se realizaron importantes cambios a la propuesta, especialmente referidos a la selección y secuenciación de los contenidos, los criterios y los indicadores de evaluación. A la vez que se identificó la necesidad de elaborar materiales curriculares complementarios a modo de guías de implementación de la propuesta curricular con especificidades para cada área sobre cómo llevar el currículo al aula (págs. 622).

Como hemos dicho con anterioridad, la propuesta elaborada no se desarrolla por años de escolarización, y menos aún por unidades didácticas, abordando exclusivamente el diseño de cada subnivel educativo. Este hecho causó perplejidad en los docentes, que para poder llevar el currículo al aula debían ahora realizar un importante trabajo en el segundo y tercer nivel de concreción curricular, desarrollando un proyecto curricular propio en el seno de la institución educativa, así como las programaciones anuales, las unidades didácticas y las programaciones de aula.

Si bien este nuevo abordaje de la concreción del currículo permite elaborar una propuesta educativa contextualizada a las necesidades e intereses de las comunidades educativas, dado que el currículo ofrece esa flexibilidad y tiene la apertura para que las instituciones educativas incorporen los elementos que estimen precisos en su situación concreta, mediante un proceso dialógico con su comunidad educativa, los docentes del magisterio ecuatoriano no estaban preparados para ese reto. Como señalan Barrera, 
Barragán y Ortega (2017): las guías curriculares y la capacitación que se ofreció al $100 \%$ del magisterio con el lanzamiento del nuevo currículo, sin embargo, no fueron suficientes y el proceso de apropiación del nuevo instrumento aún no finaliza (pág. 12).

Finalmente, la nueva propuesta sirvió de base para el desarrollo de los currículos de las 14 nacionalidades del país de acuerdo con el Modelo del Sistema de Educación Intercultural Bilingüe, que entraron en vigor en el año 2017, al igual que el Currículo Integrado de Alfabetización y las Adaptaciones curriculares para Educación Básica Superior y Bachillerato para personas con escolaridad inconclusa. Del mismo modo, durante el año 2017 y 2018 se desarrolló un proyecto con las escuelas unidocentes, bidocentes y pluridocentes del Azuay, con la participación de 23 centros y 57 docentes para establecer una metodología de trabajo en este tipo de instituciones que permitiera trabajar con la nueva propuesta curricular. Se construyeron documentos a nivel meso-curricular, como el Plan Curricular Institucional y el Plan Curricular Anual, y a nivel micro-curricular, incluyendo los Planes de Unidad Didáctica, por niveles y áreas.

\section{Conclusiones}

Las propuestas curriculares concebidas por la Autoridad Educativa Nacional en el Ecuador han evolucionado en los últimos veinte años desde los listados de contenidos de los programas de estudio de finales del Siglo XIX y principios del Siglo $\mathrm{XX}$, a estructuras complejas y bien articuladas que consideran los elementos esenciales para orientar el desarrollo de los procesos de enseñanza y aprendizaje en las aulas, incorporando, además, materiales curriculares complementarios para apoyar la acción docente y diferenciándose para atender la diversidad de nacionalidades, de las distintas realidades de las instituciones educativas y de las necesidades específicas de diferentes grupos sociales del país. Además, las nuevas propuestas han hecho 
un esfuerzo por articularse con la evaluación externa de los aprendizajes a través de estándares educativos estrechamente relacionados con el currículo, con la intención de que sirvan para sumar y no para restar a las intenciones educativas.

La definición de lo que es un contenido de aprendizaje ha evolucionado, igualmente, de una visión estrictamente conceptual o cognitiva acorde a modelos transmisivos de enseñanza a una complejidad en la que primero se agregan dimensiones procedimentales y actitudinales bajo la perspectiva de una educación integral y posteriormente se añaden criterios de desempeño orientados a delimitar las expectativas sobre la capacidad de movilización del conocimiento adquirido que los estudiantes desarrollan. Asimismo, más allá de actualización de las tradicionales áreas de conocimiento, se añaden nuevas vías para que los contenidos que la sociedad demanda ingresen al currículo, como nuevas asignaturas o temas transversales relacionados con las habilidades blandas o la convivencia. En este sentido, los contenidos del currículo han seguido una lógica acumulativa que dificulta que los docentes puedan planificar los aprendizajes previstos en los tiempos definidos para ello. Soluciones como la distinción de los contenidos básicos permiten flexibilizar las propuestas curriculares, pero no son suficientes, es preciso hacer una revisión que permita reducir los contenidos, estableciendo para ello un debate que confronte lo que se considera imprescindible incluir en el currículo desde las perspectivas disciplinares y desde la sociedad.

Por último, es evidente que desde el nivel central no se puede definir una propuesta curricular, por mucho que se trate de diversificar, que sea válida para todas las comunidades que integran nuestro sistema educativo. Las definiciones de las responsabilidades en torno a la construcción del currículo han ido variando en cada propuesta desde una mínima intervención del Estado, hasta propuestas, como la del año 2010, con un diseño cerrado y vertical, que no dejaba margen de acción a los docentes para poder vincularla 
con los intereses y necesidades de sus respectivas comunidades. En este sentido, parece razonable profundizar en una propuesta que distribuya las responsabilidades de construcción del currículo y delegue buena parte a las instituciones educativas y a los docentes que conocen los contextos en los que este se implementa; para ello es imprescindible continuar con el proceso de profesionalización docente y el desarrollo de los sistemas de apoyo.

\section{Referencias}

Barrera, H., Barragán, T., \& Ortega, G. (2017). La realidad educativa ecuatoriana desde una perspectiva docente. Revista Iberoamericana de Educación, 75(2), 9-20, ISSN: 1022-6508; e-ISSN: 1681-5653. Recuperado de: https://doi.org/10.35362/rie7522629

Bolaños, G., \& Molina, Z. (1990). Introducción al currículo. ISBN: 9977-64496-9. Costa Rica: Editorial Universitaria Estatal a Distancia - EUNED.

Calvas, V. (2010). La reforma curricular de la educación básica en ocho planteles experimentales de la zona urbana de Quito: estudio de caso realizado en el área de lenguaje y comunicación en el 3er. año de educación básica. Tesis de maestría. Quito, Ecuador: FLACSO. Recuperado de: http://hdl.handle.net/10469/2017

Coll, E. (2006a,b,c,d,e,f). Lo básico en la educación básica. Reflexiones en torno a la revisión y actualización del currículo de la educación básica. Revista Electrónica de Investigación Educativa, 8(1), 1-17, eISSN: 1607-4041. Recuperado de:

https://redie.uabc.mx/redie/article/view/139

Consejo Nacional de Educación \& Ministerio de Educación, Cultura, Deportes y Recreación (1997). Reforma curricular para la Educación Básica. Segunda edición. Quito, Ecuador: Ministerio de Educación y Cultura. Delgado, J., Vera, M., Cruz, J., \& Pico, J. (2018). El currículo de la educación básica ecuatoriana: una mirada desde la actualidad. Revista 
Cognosis, 3(4), 47-66, e-ISSN: 2588-0578. Recuperado de:

https://doi.org/10.33936/cognosis.v3i4.1462

Díaz, Á. (2015). Currículum: Entre utopía y realidad. Góndola, Enseñanza y Aprendizaje de las Ciencias, 13(2), 353-357, e-ISSN: 2346-4712.

Recuperado de: https://doi.org/10.14483/23464712.13681

Gimeno, J. (2010). ¿Qué significa el currículum? Sinéctica, (34), 11-43, ISSN: 1665-109X; e-ISSN: 2007-7033. Recuperado de:

http://www.scielo.org.mx/scielo.php?script=sci arttext\&pid=S1665$\underline{109 \times 2010000100009 \& \text { Ing }=e s \& t \operatorname{lng}=e s}$

Kreisel, M. (2016). El enfoque intercultural en el currículo de telesecundaria - Elementos para una reflexión crítica. Sinéctica, Revista Electrónica de Educación, (46), 1-18. e-ISSN: 1665-109X. Recuperado de: https://www.redalyc.org/articulo.oa?id=99843455005 Ministerio de Educación (2010a,b,c). Actualización y Fortalecimiento Curricular de la Educación General Básica. Quito, Ecuador: Ministerio de Educación del Ecuador. Recuperado de:

https://educacion.gob.ec/wp-

content/uploads/downloads/2012/08/AC 2.pdf

Ministerio de Educación (2016a). El perfil del bachiller ecuatoriano: desde la educación hacia la sociedad. ISBN: 978-9942-22-104-9. Quito, Ecuador: Ministerio de Educación del Ecuador. Recuperado de: https://educacion.gob.ec/wpcontent/uploads/downloads/2016/12/perfil-del-bachiller.pdf Ministerio de Educación (2016b). Currículo de los niveles de educación obligatoria. Ecuador: MINEDUC. Recuperado de:

https://educacion.gob.ec/wp-

content/uploads/downloads/2016/03/Curriculo1.pdf

Ministerio de Educación (2015a,b). Informe Técnico: Socialización del Currículo de la EGB 2016 a docentes y directivos del Sistema 
Educativo Nacional. Quito, Ecuador: Subsecretaría de Fundamentos Educativos Dirección Nacional de Currículo del Ministerio de Educación del Ecuador.

Morelli, S. (2019). Retoricidad y significaciones del currículum. Revista Panorâmica, 28, 10-22, e-ISSN: 2238-9210. Recuperado de:

http://revistas.cua.ufmt.br/revista/index.php/revistapanoramica/article/vi ewArticle/1050

Pascual, E. (2001). Innovación en la construcción curricular: Desafíos teóricos y prácticos en el contexto de la reforma curricular en Chile. Pensamiento Educativo, 29, 37-73, e-ISSN: 0719-0409. Recuperado de:

http://www.pensamientoeducativo.uc.cl/files/journals/2/articles/198/pub lic/198-471-1-PB.pdf

Perlo, C. (2019). La pedagogía biocéntrica: del currículum disciplinarionormativo al reencantamiento de la educación. Revista Scientific, 4(12), 68-88, e-ISSN: 2542-2987. Recuperado de:

https://doi.org/10.29394/Scientific.issn.2542-2987.2019.4.12.3.68-88

Poveda C., Díaz, G., Abendaño, A., Benalcazar, A., Araujo, S., \& Arboleda, R. (1994). Sistema Educativo Nacional del Ecuador. Quito, Ecuador: Ministerio de Educación y Cultura del Ecuador y Organización de Estados Iberoamericanos. Recuperado de:

https://www.oei.es/historico/quipu/ecuador/index.html\#sis

Sánchez, J. (2018). Currículo y vivencia. Aporía. Revista Internacional de Investigaciones Filosóficas, (16), 51-61, e-ISSN: 0718-9788. Recuperado de: https://doi.org/10.7764/aporia.16.663

Stabback, P. (2016). Qué hace a un currículo de calidad. Código del documento: IBE/2016/WP/CD/02. Suiza: Oficina Internacional de Educación de la UNESCO. Recuperado de: https://unesdoc.unesco.org/ark:/48223/pf0000243975 spa.locale=es 


\section{Miguel Ángel Herrera Pavo \\ e-mail: miguel.herrera.p@uasb.edu.ec}

Nacido en Sevilla, España, el 30 de octubre del año 1970.

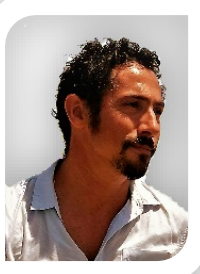

Psicopedagogo y Doctor en Sociedad de la Información y el Conocimiento; Experiencia como gestor, profesor e investigador en diversas instituciones públicas y privadas, nacionales e internacionales, en los campos de la evaluación del currículo, del e-learning, de la educación bilingüe intercultural, de la formación del profesorado, de la inclusión educativa y del género en educación; Docente del Área de Educación de la Universidad Andina Simón Bolívar (UASB), sede Ecuador; y docente colaborador en el Máster de Educación y TIC de la Universitat Oberta de Catalunya (UOC). 


\section{María Gladys Cochancela Patiño \\ e-mail: maria.cochancela@unae.edu.ec}

Nacida en Cuenca, Ecuador, el 27 de abril del año 1967.

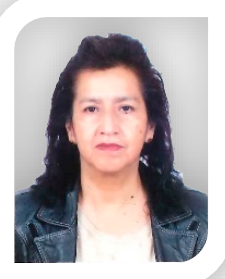

Docente-investigadora de la Universidad Nacional de Educación (UNAE) en Ecuador; Formación académica en Educación Básica y Gestión Educativa; Experiencia como docente, directora y analista en Educación Inicial y Básica, como profesora-orientadora en el Instituto Superior Pedagógico "Ricardo Márquez Tapia"; y como asesora educativa, realizando acompañamiento pedagógico en las provincias ecuatorianas de Azuay, Cañar y Morona Santiago; Directora del proyecto de investigación en la línea de acción de currículo y pedagogía.

El contenido de este manuscrito se difunde bajo una Licencia de Creative Commons ReconocimientoNoComercial-Compartirlgual 4.0 Internacional 